\title{
Late Pulmonary Embolism after COVID-19 Pneumonia despite Adequate Rivaroxaban Treatment
}

\author{
Giuseppe Di Tano ${ }^{1}$, Luigi Moschini ${ }^{1}$, Marco Loffi ${ }^{1}$, Sophie Testa ${ }^{2}$, Gian Battista Danzi ${ }^{1}$ \\ ${ }^{1}$ Division of Cardiology, Ospedale di Cremona, Italy \\ ${ }^{2}$ Haemostasis and Thrombosis Center, Ospedale di Cremona, Italy
}

Received: 09/06/2020

Accepted: $13 / 06 / 2020$

Published: 18/06/2020

How to cite this article: Di Tano G, Moschini L, Loffi M, Testa S, Danzi GB. Late pulmonary embolism after COVID-19 pneumonia despite adequate rivaroxaban treatment. EJCRIM 2020;7: doi:10.12890/2020_001790.

Conflicts of Interests: The Authors declare that there are no competing interests.

This article is licensed under a Commons Attribution Non-Commercial 4.0 License

\section{ABSTRACT}

Introduction: SARS-CoV-2 infection may predispose patients to thrombotic disease. Patients with COVID-19 pneumonia who are receiving non-vitamin $\mathrm{K}$ antagonists or direct oral anticoagulants for chronic disease are usually switched to heparin treatment during hospitalization. However, information about the most appropriate antithrombotic therapy after the acute infection phase is lacking.

Case Description: We report the case of a patient with chronic atrial fibrillation who was recently hospitalized for severe COVID-19 pneumonia. Four weeks after discharge he experienced an episode of an acute pulmonary embolism while on rivaroxaban therapy with adequate drug plasma levels, and in the absence of strong predisposing risk factors.

Conclusion: This case highlights the risk of thrombotic complications after COVID-19 infection, raises some concern about their underlying mechanisms, and supports the use of effective anti-thrombotic therapy.

\section{LEARNING POINTS}

- COVID-19 infection is associated with frequent thrombotic events.

- A pro-coagulative status could be triggered by the persistent inflammatory phase of the infection despite anticoagulation.

- Adequate antithrombotic therapy is necessary for the prevention of acute and later thrombotic complications and needs close monitoring.

\section{KEYWORDS}

Viral diseases, pneumonia, thrombosis, pulmonary embolism, rivaroxaban

\section{INTRODUCTION}

Emerging evidence suggests that the coagulation function is significantly deranged during SARS-CoV-2 (COVID-19) infection and may predispose to arterial and venous thrombotic complications due to excessive inflammation, platelet activation, endothelial dysfunction and stasis $^{[1]}$. During the acute infection phase, patients chronically managed with antithrombotic agents (non-vitamin K antagonists (VKAs) and direct oral anticoagulants (DOACs)) are usually switched to alternative parenteral antithrombotic medications such as low molecular weight heparin $(\mathrm{LMWH})^{[2,3]}$. However, information about the most appropriate antithrombotic therapy after the acute infection period is lacking. We report the case of a patient with chronic atrial fibrillation (AF) who was recently hospitalized for severe COVID-19 pneumonia. Four weeks after discharge he experienced an episode of acute pulmonary embolism (APE) while on rivaroxaban therapy at the appropriate dosage and with adequate drug plasma levels. 


\section{CASE DESCRIPTION}

A 79-year-old man was hospitalized for severe bilateral COVID-19 pneumonia after 10 days of fever and dyspnoea. He had a history of hypertension and of long-standing AF for which he was on rivaroxaban therapy (20 mg per day). During hospitalization, DOAC was switched to $\mathrm{LMWH}$ at a therapeutic regimen (enoxaparin $100 \mathrm{IU} / \mathrm{kg}$ bid) and administered with hydroxychloroquine, lopinavir/ritonavir, empiric antimicrobial treatment and supplemental oxygen to maintain $\mathrm{SpO} 2$ in the $93-96 \%$ range. Coagulation parameters were in the normal range. The patient's clinical condition improved and the hospital course was uneventful. He was discharged after 19 days with the indication to resume rivaroxaban treatment together with the anti-hypertensive drug losartan (100 mg per day).

Two weeks later he suddenly complained of severe respiratory distress with cough and chest pain. In the emergency department, the electrocardiogram confirmed the presence of AF with a ventricular rate of $110 \mathrm{bpm}$. Laboratory tests revealed a normal complete blood count with increased values of hs-troponin I (230.2 ng/l, normal value <34.2), D-dimer (3.99 $\mu \mathrm{g} / \mathrm{ml}$, normal value <5.00), C-reactive protein (47 mg/l, normal value <5.00), fibrinogen ( $376 \mathrm{mg} / \mathrm{dl}$, normal value <200), lactate dehydrogenase (325 U/l, normal value <248) and ferritin (353 ng/ml, normal value 21-246). Transthoracic echocardiography showed normal size and function of the right ventricle with a mild increase in pulmonary pressures $(40 \mathrm{mmHg})$. Computed tomography pulmonary angiography showed APE involving the lateral and posterior basal segmental branches of the right pulmonary artery and the posterior basal segmental branch of the left pulmonary artery (Fig. $1 A, B)$. The ground-glass opacities documented on computed tomography scanning during the first hospitalization had evolved into areas of consolidation and of interlobular and intralobular septal thickening (Fig. 1C,D). The patient stated that he was taking the drugs regularly. The rivaroxaban plasma level was promptly measured (20 hours after last administration the day before hospitalization) and was 68 ng/ $\mathrm{ml}$ (normal value in the absence of the drug is $<25 \mathrm{ng} / \mathrm{ml}$ ) with an elevated PT INR of 2.16 and aPTT of 1.45 (normal value $<1.2$ ). Based on these findings, the patient was switched to $\mathrm{LMWH}$ at a full dose regimen (100 IU/kg bid). Lower limb compression ultrasonography was negative for venous thromboembolism (DVT). The clinical course was uneventful and the patient was discharged home 9 days later on therapeutic doses of LMWH.

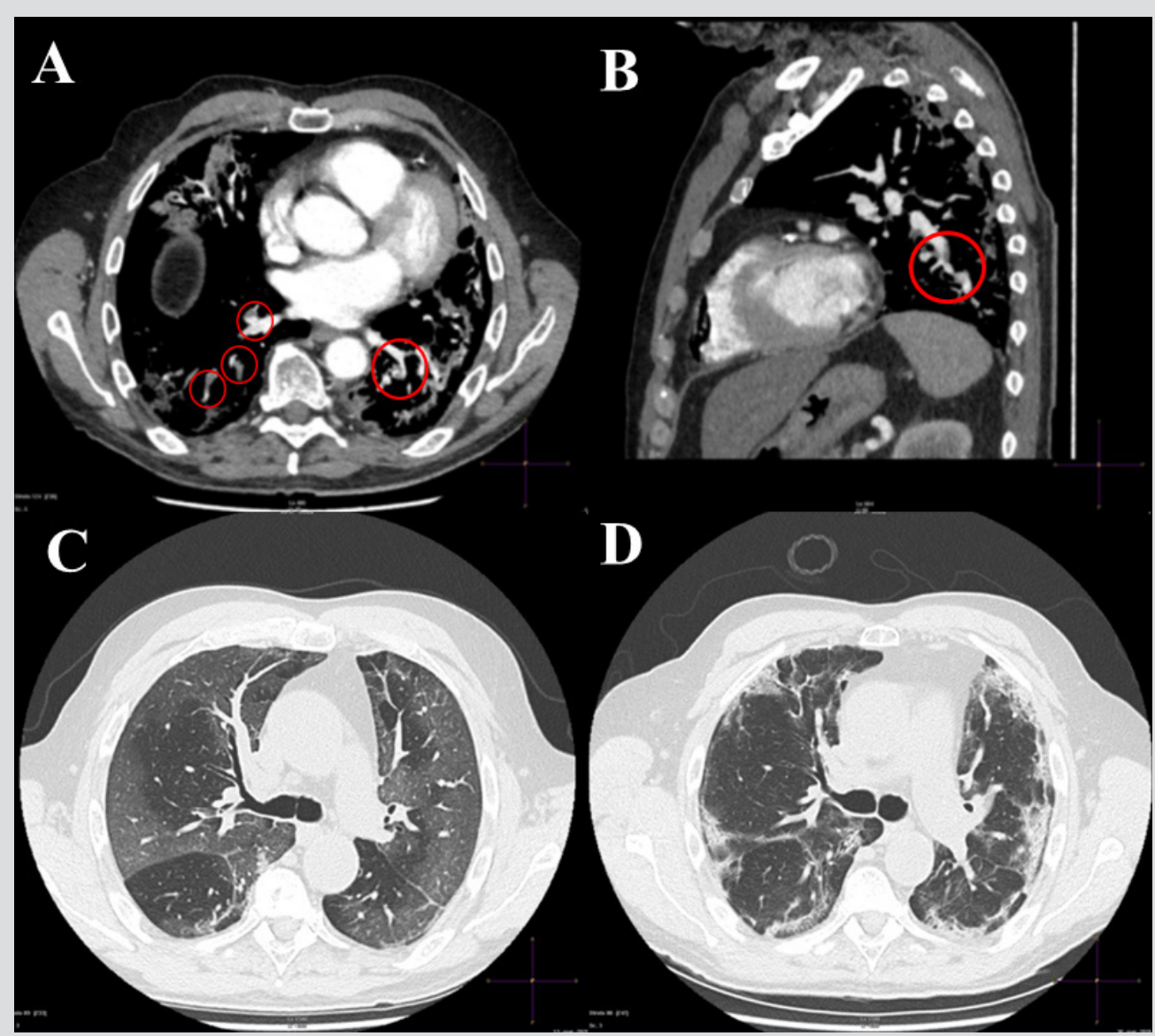

Figure 1. (A) CT pulmonary angiography (axial view) showing filling defects involving the lateral and posterior basal segmental branches of the right pulmonary artery (open circles) and the posterior basal segmental branch of the left pulmonary artery (open circle). (B) CT pulmonary angiography (sagittal view) depicting a filling defect localized on the inferior lobar branch of the left pulmonary artery (open circle). (C) Chest CT scan (axial view) during the first hospitalization showing extensive areas of ground-glass opacities involving both lung parenchymas. (D) Control chest CT scan during rehospitalization showing areas of consolidations and interlobular and intralobular septal thickening 


\section{DISCUSSION}

Thrombotic events in patients who are on anticoagulation therapy are uncommon. In the PREFER Registry, among patients receiving chronic anticoagulation for AF, the DVT recurrence rate was $3.9 \%$ with an overall incidence of PE of $1.3 \%{ }^{[4]}$. Furthermore, only one recurrent episode of DVT (1.7 events per 100 patient-years) was documented in the more recent Dresden NOAC Registry ${ }^{[5]}$. The main reason for a breakthrough event is underlying disease and sub-therapeutic drug level.

During COVID-19, patients on oral anticoagulation therapy are exposed to the risk of an unstable therapeutic level due to concomitant therapies. For this reason, in order to prevent bleeding complications, oral anticoagulants are replaced with heparins during hospitalization ${ }^{[3]}$. Information about the most appropriate antithrombotic therapy after the acute infection phase is lacking. However, recent suggestions recommend DOACs should be preferred over VKAs because of their better safety with fixed dosing without the need for laboratory monitoring and to limit patient contact with healthcare services, all characteristics of particular relevance for outpatient management in this pandemic ${ }^{[2,4]}$.

Our case raises some concerns about the appropriateness of DOAC treatment in the subacute phase of COVID-19 infection for patients who need oral anticoagulation treatment. Despite correct adherence to rivaroxaban therapy with the expected plasma levels, the patient developed an acute pulmonary thrombotic event after the interstitial pneumonia had apparently, but not completely, resolved. Indeed 2 weeks after discharge, inflammatory markers such as C-reactive protein, lactate dehydrogenase, fibrinogen and ferritin were still high.

We suppose that the segmental APE event in our patient, without evidence of DVT, may be closely related to the pro-coagulative status triggered by the persistent inflammatory phase of the SARS-CoV-2 infection ${ }^{[1]}$. Autopsy specimens from COVID-19 patients showed that pulmonary arteries at the hilum were free of thromboemboli, whereas small-vessel thrombus formation was seen in the periphery of the lung parenchyma in the majority of cases, supporting the concept of a hypercoagulative status, and a high frequency of pulmonary micro-thrombosis ${ }^{[6,7]}$. SARS-CoV-2 infection facilitates the induction of endotheliitis in several organs as a direct consequence of viral involvement (as noted with the presence of viral bodies) and of the host inflammatory response ${ }^{[7]}$. These observations could explain the inefficacy of a direct inhibitor of activated factor Xa such as rivaroxaban, which is active only on the coagulation cascade, while the antiinflammatory and immunomodulatory properties of heparin, which can stabilize the endothelium, could be more helpful for this specific situation as reported ${ }^{[8]}$.

In conclusion, our case highlights the risk of later thrombotic complications after COVID-19 infection despite appropriate oral anticoagulant therapy and raises some concern about the underlying mechanism. In patients on chronic oral anticoagulant therapy, discharged after hospitalization for acute COVID-19, close monitoring is recommended, and LMWH replacement should be considered only until evidence of complete remission of disease.

\section{REFERENCES}

1. Bikdeli B, Madhavan MV, Jimenez D, Chuich T, Dreyfus I, Driggin E, et al. COVID-19 and thrombotic or thromboembolic disease: implications for prevention, antithrombotic therapy, and follow-up. J Am Coll Cardiol 2020;75(23):2950-2973.

2. Thachil J, Tang N, Gando S, Falanga A, Cattaneo M, Levi M, et al. ISTH interim guidance on recognition and management of coagulopathy in COVID-19. J Thromb Haemost 2020;18(5):1023-1026.

3. Testa S, Paoletti O, Giorgi-Pierfranceschi M, Pan A. Switch from oral anticoagulants to parenteral heparin in SARS-CoV-2 hospitalized patients. Intern Emerg Med 2020 April 15;1-3. doi: 10.1007/s11739-020-02331-1 [Epub ahead of print].

4. Cohen AT, Gitt AK, Bauersachs R, Fronk EM, Laeis P, Mismetti P, et al. The management of acute venous thromboembolism in clinical practice. Results from the European PREFER in VTE Registry. Thromb Haemost 2017;117(7):1326-1337.

5. Beyer-Westendorf J, Förster K, Pannach S, Ebertz F, Gelbricht V, Thieme C, et al. Rates, management, and outcome of rivaroxaban bleeding in daily care: results from the Dresden NOAC Registry. Blood 2014;124:955-962.

6. Fox SE, Akmatbekov A, Harbert JL, Li G, Brown JQ, Vander Heide RS. Pulmonary and cardiac pathology in COVID-19: the first autopsy series from New Orleans. medRxiv Preprint 2020. doi: https://doi.org/10.1101/2020.04.06.20050575.

7. Varga Z, Flammer AJ, Steiger P, Haberecker M, Andermatt R, Zinkernagel AS, et al. Endothelial cell infection and endotheliitis in COVID-19. Lancet 2020;395(10234):14171418.

8. Thachil J. The versatile heparin in COVID-19. J Thromb Haemost 2020;18:1020-1022. 